\title{
Immunogenicity of a universal HIV-1 vaccine vectored by DNA, MVA and CHADV-63 in a Phase I/IIA clinical trial
}

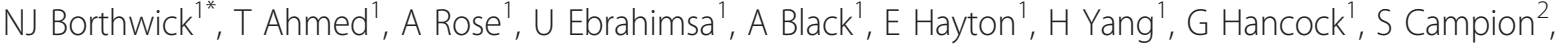 \\ N Frahm ${ }^{3}$, S Colloca ${ }^{4}$, A Nicosia $^{4}$, A McMichael $^{5}$, L Dorrell $^{5}$, T Hanke $^{1}$
}

From AIDS Vaccine 2012

Boston, MA, USA. 9-12 September 2012

\section{Background}

The major challenge facing both antibody and $\mathrm{T}$ celleliciting vaccines against HIV-1 is the extreme variability of the HIV-1 genome: a successful vaccine has to effectively target diverse HIV-1 strains circulating in the population and then must deal with ongoing virus escape in infected individuals. To address these issues, we assembled a vaccine immunogen HIVconsv from the functionally most conserved regions (not epitopes) of the HIV-1 proteome.

\section{Methods}

A gene coding for the HIVconsv immunogen was inserted into plasmid DNA (D), modified vaccinia virus Ankara (MVA; M) and non-replicating adenovirus of a chimpanzee origin ChAdV-63 (C). Currently, combined heterologous prime-boost regimens of these vaccines, namely CM, DDDCM and DDDMC, are being evaluated in a phase I/IIa trial HIV-CORE002 in healthy HIV-1/2negative volunteers in Oxford.

\section{Results}

Preliminary data indicate that the vaccines are well tolerated and show high immunogenicity. Following the CM regimen, vaccine-induced $\mathrm{T}$ cell frequencies reached a median of 5150 (range 1475 to 16495 ) SFU $/ 10^{6}$ PMBC ex vivo one week post MVA vaccination. DNA priming increased subsequent $\mathrm{T}$ cell responses to ChAdV-63 vaccination (median: C 577 and DDDC 1328 SFU/10 6 PBMC) and ELISpot responses again peaked 1 week following MVA (median 4500; range 2260-7960 SFU/106 PBMC).
Matrix analyses of the participants following CM vaccination showed that $\mathrm{T}$ cells responded to a range of peptides across the length of HIVconsv. The CM regimen elicited IFN- $\gamma$ in both CD4+ and CD8+ T cell subsets and polyfunctional (IFN- $\gamma$ \& TNF- $\alpha$ ) responses to HIVconsv peptides.

\section{Conclusion}

Presented data will be very much work in progress. Nevetheless, the HIVconsv vaccines have so far induced $\mathrm{T}$ cell responses superior to other HIV-1 vaccine candidates tested to date. ChAdV-63 is the first adenovirus of chimp origin delivering an HIV-1-derived immunogen that has reached the clinic.

The work is supported by Medical Research Council UK.

\section{Author details}

${ }^{1}$ University of Oxford, Oxford, UK. ${ }^{2}$ University of Oxford, Human Immunology Unit, Oxford, UK. ${ }^{3}$ HIV Vaccine Trials Network, University of Washington, Seattle, USA. ${ }^{4}$ Okairos, Rome, Italy. ${ }^{5}$ Oxford University, Human Immunology Unit, Oxford, UK.

Published: 13 September 2012

\footnotetext{
doi:10.1186/1742-4690-9-S2-P118

Cite this article as: Borthwick et al:: Immunogenicity of a universal HIV-1 vaccine vectored by DNA, MVA and CHADV-63 in a Phase I/IIA clinical trial. Retrovirology 2012 9(Suppl 2):P118.
}

Tniversity of Oxford, Oxford, UK

Full list of author information is available at the end of the article 